\title{
Treatment of intralesional corticoisteroid-induced cutaneous atrophy with hyaluronic acid filling
}

\section{Introduction}

Frontal fibrosing alopecia (FFA) is an acquired scarring alopecia with histopathologic finding identical to lichen planopilaris (LPP). Clinically FFA is characterized by hair loss in the line of frontal hair implantation, sometimes associated with loss of eyebrows and other areas and perifollicular erythema. ${ }^{1}$ Around the upper portion of the hair follicle there is an inflammatory lymphocytic infiltration that usually begins before the destruction of the follicles. ${ }^{2}$ First line treatments are 5 - $\alpha$-reductase inhibitors and intralesional corticosteroids. Several adverse reactions secondary to the injection of corticosteroids have been described (pigment alteration, pain, infection, panniculitis, atrophy and hemorrhage). ${ }^{3}$

\section{Case report}

A 46years-old woman presented FFA consistent with a progressive alopecia localized to the frontal and temporal hairline as a band with atrophic and shiny skin. Follicular keratinization and perifollicular erythema were present. We prescribed treatment with oral dutasteride $0,5 \mathrm{mg}$ daily combined with $5 \%$ minoxidil lotion. We infiltrated intralesional corticosteroids along the line of frontal hair implantation. Injections were performed with triamcinolone acetonide $0,1 \mathrm{ml}$ diluted

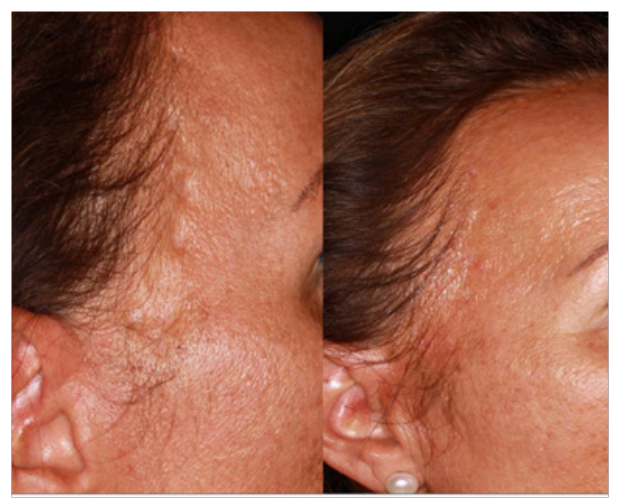

Figure I FFA, right side of the face. Before and after hyaluronic acid filling.

\section{Discussion}

Frontal fibrosing alopecia is a disease with possible multifactorial etiology, genetic, immune, hormone and environmental. Intralesional corticosteroid (Cs) is useful when administered in the initial stages of the disease, to avoid the damage of the lichenoid inflammatory cells to the hair follicle. ${ }^{4}$ Local cutaneous atrophy is an adverse reaction to Cs injection and is determined by solubility and concentration of the Cs solution and the depth and anatomic area of injection. Glucocorticoids act on subcutaneous tissue by inhibiting fibroblasts and decreasing synthesis of collagen, elastin and mucopolysaccharides. In addition, skin thinning is believed to be produced due to vasoconstriction and deposits of insoluble corticosteroid crystals for a long time with decreased type I collagen and glycosaminoglycan synthesis. These reactions are more frequent with triamcinolone compared with highsolubility Cs such as methylprednisolone and betamethasone.

Normal saline infiltration and fat injection has been reported as potential therapies of Cs-induced cutaneous atrophy. ${ }^{5}$ The mechanism de action of saline solution appears to be through the re-suspension

\author{
Volume 2 Issue 4 - 2018 \\ Irene García-Morales, ' José Luis Marínez- \\ Amo, ${ }^{2}$ Sergio Vañó-Galván ${ }^{3}$ \\ 'Department of Dermatology, Hospital Universitario Virgen de \\ Valme, Spain \\ ${ }^{2}$ Department of Dermatology, Hospital de Poniente, Spain \\ ${ }^{3}$ Department of Dermatology, Hospital Universitario Ramón y \\ Cajal, Spain
}

Correspondence: Irene García-Morales MD, Hospital Universitario de Valme, Ctra de Cádiz Km548, 94I00I, Sevilla (España),Spain,Tel 90250506I,Email irenegmorales@gmail.com

Received: March 13, 2018| Published: July 12, 2018

in 0,4 lidocaine $1 \%$. We administered $0,03 \mathrm{ml}$ per point following a headband distribution. The patient attends a week later for the appearance of depressions located in sites of corticosteroid injections. We refilled the cutaneous atrophy by infiltrating hyaluronic acid (Belotero Soft ${ }^{\mathbb{R}}$ ) with subcutaneous micro depot technic, obtaining excellent results in only one session (Figures 1) (Figures 2).

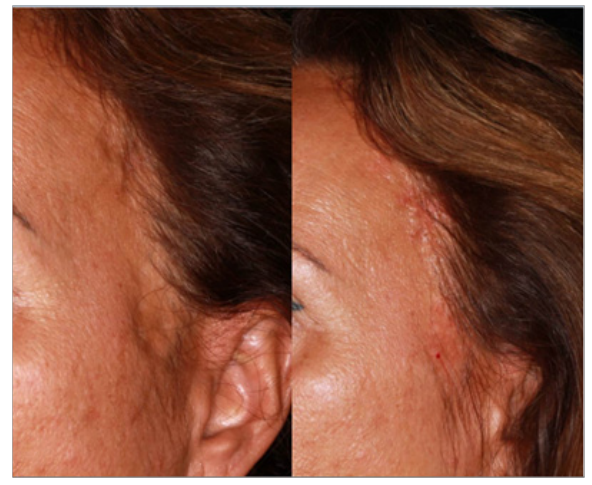

Figure 2 FFA, left side of the face. Before and after hyaluronic acid filling.

and re-dissolve of the steroid crystals present in the tissues. Fat injection seems to improve cutaneous atrophy by adipose-derived stem cells and increase of vasculogenesis.

\section{Conclusion}

The mechanism of Cs-induced cutaneous atrophy seems to be due to several factors, mainly decreased type I collagen and glycosaminoglycan synthesis and vasoconstriction. Generally, cutaneous atrophy resolve spontaneously in a few months but patients often ask for a solution before spontaneous resolution. We refilled the cutaneous atrophy by infiltrating hyaluronic acid obtaining excellent results in a simple session with long-term improvement. In our experience, hyaluronic acid infiltration is a simple, fast and safe option.

\section{Acknowledgements}

None. 


\section{Conflict of interest}

The author declares no conflict of interest.

\section{References}

1. Rallis E, Stamatis G, Eleftheria C. Frontal fibrosing alopecia: to treat or not to treat? J Cutan Med Surg. 2010;14(4):161-166.

2. Moreno-Ramirez D, Ferrandiz L, Camacho FM. Diagnostic and therapeutic assessment of frontal fibrosing alopecia. Actas Dermosifiliogr. 2007;98(9):594-602.
3. Goldman L. Reactions following intralesional and sublesional injections of corticosteroids. JAMA. 1962;182(6):613-616.

4. Nusrat Banka1, Thamer Mubki, Marry Jo Kristine Bunagan. Frontal fibrosing alopecia: a retrospective clinical review of 62 patients with treatment outcome and long-term follow-up. International Journal of Dermatology. 2014;53(11):1324-1330.

5. Shumaker P, Rao J, Goldman M. Treatment of local, persistent cutaneous atrophy following corticosteroid injection with normal saline infliltration. Dermatol Surg. 2005;31(10):1340-1343. 